Mots. Les langages du politique

$91 \mid 2009$

Que devient le pamphlet?

Ruth Wodak, The Discourse of Politics in Action. Politics as Usual

Dominique Maingueneau

\title{
OpenEdition
}

Journals

Édition électronique

URL : https://journals.openedition.org/mots/19305

DOI : $10.4000 /$ mots. 19305

ISSN : 1960-6001

Éditeur

ENS Éditions

Édition imprimée

Date de publication : 30 novembre 2009

Pagination : 135-140

ISBN : 9782847881820

ISSN : 0243-6450

Référence électronique

Dominique Maingueneau, "Ruth Wodak, The Discourse of Politics in Action. Politics as Usual », Mots. Les langages du politique [En ligne], 91 | 2009, mis en ligne le 30 novembre 2011, consulté le 24 avril 2022. URL : http://journals.openedition.org/mots/19305 ; DOI : https://doi.org/10.4000/mots. 19305 


\section{The Discourse of Politics in Action. Politics as Usual}

Ruth Wodak

2009, New York, Palgrave Macmillan, 252 p.

La linguiste autrichienne Ruth Wodak est surtout connue comme l'un des auteurs majeurs de la Critical Discourse Analysis, dont elle est en outre l'une des figures fondatrices à côté de chercheurs tels que Teun Van Dijk ou Norman Fairclough ${ }^{1}$. À la différence de chercheurs comme Van Dijk, qui met l'accent sur la dimension cognitive des processus idéologiques, elle y développe une approche centrée sur la contextualisation sociale des discours. Après avoir longtemps enseigné à l'université de Vienne, elle travaille depuis 2004 à l'université de Lancaster, en Grande-Bretagne, où elle est titulaire de la chaire de Discourse Studies. Ce passage du continent au monde anglo-saxon est symptomatique de son attitude, qui consiste à intégrer des apports très divers.

Elle s'est fait connaitre en particulier par ses travaux sur l'antisémitisme et l'extrême droite dans son pays, ce qui lui a valu le prestigieux prix Wittgenstein en 1996. Mais son champ d'activité est beaucoup plus large, comme le montre son dernier livre, The Discourse of Politics in Action. Politics as Usual, qui vient de paraitre chez Palgrave Macmillan et qui traite des activités des députés au Parlement européen. Cet intérêt pour ce sujet n'est pas nouveau; elle avait déjà participé à une recherche interdisciplinaire, dont les résultats ont été publiés en 2000 (Muntigl, Weiss, Wodak), sur une commission d'experts des institutions européennes (Commission's Competitiveness Advisory Group). L'ouvrage qui vient de paraitre clôture de longues années de recherches interdisciplinaires sur ce terrain.

Le lecteur français pourra sembler de prime abord dérouté par une démarche bien éloignée des tendances dominantes dans l'hexagone. Il existe en effet en France comme dans beaucoup de pays une certaine répartition du travail, selon laquelle l'étude du politique est divisée entre ceux qui se préoccupent de textes et de doctrines, et ceux, nécessairement moins nombreux, qui proposent des approches nettement «ethnologiques» : il suffit de songer aux travaux de Marc Abélès sur le Parlement européen (1992) ou à ceux d'Irène Bellier (1993) qui a d'ailleurs elle aussi étudié le Parlement européen. Or le livre que nous propose Ruth Wodak s'efforce d'unifier ces deux types d'approche, de raisonner à la fois en termes de discours et en termes d'anthropologie. Ce que l'auteure elle-même caractérise comme "qualitative interdisciplinary research" (p. 26).

1. On notera que la revue Semen vient de publier un numéro spécial sur la «Critical Discourse Analysis» ( $\mathrm{n}^{\circ} 27$, avril 2009). Il contient un article de Ruth Wodak très significatif de sa démarche : «Pragmatique et analyse de discours critique. Un exemple d'une analyse à la croisée des disciplines» (p. 97-126). 
Le choix d'une démarche ouverte sur de multiples champs de savoir est évidemment à la mesure de l'objet d'étude, la politique au quotidien, la «politique du couloir» (pour reprendre une expression chère à l'auteure) dans un lieu, le Parlement européen, où le rapport à la politique nationale ne peut qu'être indirect. L'une des caractéristiques majeures de cette institution est en effet que le personnel politique est prélevé dans divers espaces nationaux mais qu'une fois à Bruxelles, il est pris dans une communauté qui fonctionne selon des règles spécifiques auxquelles les catégories classiques de l'analyse politique ne peuvent pas s'appliquer. Pour autant, la recherche de Ruth Wodak ne vise pas à replier cette communauté sur ses rites; elle s'en sert plutôt comme d'un miroir grossissant pour réfléchir sur la nature de l'activité des hommes politiques.

Il s'agit en effet d'analyser ce que font au quotidien les professionnels de la politique, désignation périphrastique ici mieux adaptée que des termes comme «politiciens» ou "personnel politique». Elle s'en explique dans la préface du livre :

I finally decided to dedicate an entire book to investigate the political profession: to find out "how politics is done", "what politicians actually do", and "what the media convey about how politics is done". Moreover, I also wanted to probe the implications of the public's lack of knowledge about the behind-the-scene reality of "politics as usual" in an era of politics that many characterize in terms of an increasing and widespread disenchantment with politics, depoliticization and the so-called "democratic deficit". [C'est nous qui soulignons en romain] (p. xii)

L'accent est ainsi mis sur ce que les professionnels de la politique font en réalité, c'est-à-dire sur l'écart entre les représentations communes et l'activité effective, entre la scène et les coulisses. Ce changement d'espace s'accompagne d'un changement d'ordre de réalité qui est aussi un changement d'ordre de pratiques discursives : non pas les discours au sens usuel, les performances officielles, mais les multiples pratiques verbales et non verbales qui les rendent possibles, et dont le lien avec «la politique» au sens habituel du mot semble souvent ténu.

Le chapitre I (“Doing politics"), comme on peut s'y attendre, présente les concepts et les approches théoriques sur lesquels repose le travail. L'accent est mis sur l'opposition qui traverse tout le livre entre frontstage et backstage, la scène et les coulisses, qui est au cœur de l'activité politique. L'une des forces de la démarche choisie est de combiner des approches micro- et macrosociologiques, Goffman et Bourdieu (habitus, capital, marché). Ruth Wodak prête également une attention particulière au concept de "communauté de pratique» (développé par Lave et Wenger, 1991), qui lui permet d'articuler l'habitus et le self. Ce concept a une portée très large, puisqu'il désigne le processus d'apprentissage et les pratiques partagées qui émergent et évoluent quand un groupe d'individus interagissent pour parvenir collectivement à un certain résultat. 
Le chapitre II ("The [ir]ationality of politics") est plus particulièrement centré sur le cadre théorique spécifique dont se réclame l'auteure, qu'elle nomme "Discourse-Historical Approach", qui s'inscrit dans le cadre plus large de la Critical Discourse Analysis. Pour elle, cette approche "provides a vehicle for looking at latent power dynamics and the range of potentials in agents, because it integrates and triangulates knowledge about historical sources and the background of the social and political fields within which discursive events are embedded" (p.38). Comme dans le modèle de Norman Fairclough, ce qui est placé au centre, c'est l'intégration des pratiques discursives dans des cadres sociohistoriques. Quatre niveaux (layers) de contexte sont pris en compte (p.38):

- les relations intertextuelles et interdiscursives entre énoncés, genres et discours;

- les variables sociologiques extralinguistiques;

- l'histoire et l'archéologie des textes;

- les cadres institutionnels du contexte spécifique d'une situation déterminée.

C'est au chapitre III (“'Politics as usual' on the 'European stage': constructing and performing 'European identities'”) que commence l'étude empirique proprement dite. Le Parlement européen constitue un espace privilégié quand il s'agit, comme le fait Ruth Wodak, de mettre l'accent sur les identités multiples dont participent les députés, qui sont en outre tiraillés entre diverses conceptions de la Communauté européenne. Ce chapitre ne se contente pas d'exposer l'organigramme du Parlement, mais il insiste sur l'architecture, le décor, les caisses de documents sans cesse remplies, vidées, transportées, les interminables couloirs qu'arpentent des députés chargés de dossiers. La «communauté de pratique» qui rassemble les députés entre constamment en tension avec les identités multiples de ses membres, engagés dans des activités verbales très diverses : conversations avec les assistants, feuilletage de documents à peine lus, réunions d'experts de toutes sortes... La recherche prend appui sur un certain nombre d'entretiens avec des députés, dont des extraits significatifs sont étudiés à travers des marquages comme les métaphores, les topoi argumentatifs et les évaluations.

Le chapitre suivant ("One day in the life of an MEP") est sans doute le plus stimulant pour le lecteur, puisqu'il présente une étude de cas. La perspective se fait ici franchement ethnographique. Le député étudié, désigné par le nom «Hans», a accepté de porter un microphone et un magnétophone, de façon à pouvoir être suivi à travers la série des interactions de sa journée, qui commence à huit heures par un entretien avec son assistant et s'achève par une conférence au "Club socio-démocrate» du Parlement. La vie d'un député européen ne semble pas de tout repos : se lever tôt, se coucher tard, cheminer plus de $10 \mathrm{~km}$ par jour à l'intérieur du Parlement en transportant une 
impressionnante quantité de documents... En associant intimement pratiques verbales et non verbales, on saisit sur le vif le mélange d'ordre et de désordre de l'activité des députés. Cette dialectique de l'ordre et du désordre constitue d'ailleurs un des leitmotiv de l'ouvrage. Ruth Wodak montre que ces acteurs disposent de compétences qui leur permettent d'être à la fois immergés dans des situations singulières et de négocier entre leurs diverses identités, sans perdre complètement de vue les grandes stratégies qui sont censées gouverner leurs actions. Cette étude confirme l'une des idées clés du livre de l'auteure: "When following Hans throughout his entire day, it became apparent that he constructs his multiple identities in ever new ways, depending on the specific context." (p.151)

Le chapitre V ("Everyday politics in television: fiction and/or reality") peut surprendre de prime abord, puisqu'il abandonne abruptement les activités des députés du Parlement européen pour s'intéresser aux fictions télévisées qui prétendent donner accès aux coulisses de la vie politique. La plus connue d'entre elles est la série américaine The Left Wing, qui prétend montrer ce qui se passe à la Maison Blanche, en particulier quand le président des États-Unis est aux prises avec des groupes qui menacent la sécurité du pays. Regardée par 14 millions d'Américains, cette série est diffusée dans de nombreux pays. L'étude de Ruth Wodak permet de voir en quoi ces fictions télévisées divergent de la réalité. Mais sa portée va bien au-delà : comme le laissait déjà entendre l'opposition centrale du livre entre backstage et frontstage, la question de la politique et celle de la mise en scène sont indissociables. Étant donné le rôle stratégique que jouent les médias, et en particulier la télévision, dans le monde contemporain pour normer les représentations collectives, l'irruption d'un tel chapitre n'a rien de surprenant. Pas de société qui ne "fictionnalise» le politique, et pas de professionnels de la politique qui ne s'appuient sur ces fictions pour se mettre eux-mêmes en scène. Les conclusions de l'analyse de Ruth Wodak sont conformes à ce que l'on pouvait attendre : dans ces fictions, la complexité des processus de prise de décision est réduite à des situations élémentaires, centrées sur des héros charismatiques. Plus fondamentalement, l'activité politique dans ce type de production de masse est structurée selon des contraintes narratives canoniques, alors que la pratique de la politique au quotidien montre une multitude d'activités enchevêtrées qui ne se laissent pas ramener à des schémas cognitivement satisfaisants. La fonction compensatoire de tels feuilletons, dès lors, ne fait aucun doute : ils proposent aux téléspectateurs des univers où le politique est transparent, maitrisable.

La conclusion du livre ("Order or disorder - Fiction or reality? The implications of 'power and knowledge management' on 'politics as usual'") s'efforce de lier les conclusions des deux branches de l'exposé : l'étude des activités des députés européens et celle de la fiction télévisée. C'est l'occasion pour l'auteure d'insister sur le fossé qui sépare les deux univers. L'activité poli- 
tique au quotidien montre un apparent chaos, qui est en fait structure "by the amount and quality of knowledge shared by specific communities of practice" (p.187). Comme une telle réalité n'est pas accessible à ceux qui sont étrangers à cette communauté, l'écart avec les attentes et les représentations des profanes ne fait que s'accroitre. Ce chapitre conclusif est aussi l'occasion de dresser un bilan d'ordre épistémologique sur le dispositif interdisciplinaire qui a été mobilisé : de l'ethnographie à la linguistique en passant par Goffman, Bourdieu ou Max Weber. Mais le dernier mot est pour le «déficit démocratique » qu'implique le fonctionnement effectif de la politique et pour les fictions, dont le rôle est à la fois de compenser et de renforcer les frustrations des citoyens ordinaires. Pour sortir de ce cercle vicieux, Ruth Wodak ne propose pas de solutions; elle espère néanmoins que le type de recherche qu'elle mène peut contribuer à rendre moins opaques les activités de ceux dont les décisions engagent de multiples aspects de notre propre vie de tous les jours.

Nous n'avons pu donner qu'une idée très vague d'un livre extrêmement riche, qui s'efforce constamment de faire comprendre au lecteur la diversité des facteurs qui interagissent à tous les niveaux de l'activité discursive des professionnels de la politique.

L'un des traits les plus remarquables de cette recherche est sans nul doute qu'elle est ouverte sur le plan théorique et méthodologique. En analyse du discours, on a coutume d'opposer les approches microsociologiques qui dominent dans les travaux interactionnistes américains et les approches macrosociologiques qui seraient dominantes en Europe. Se tenant au plus près de son terrain, Ruth Wodak se joue allègrement de ce type d'opposition, comme de divers autres. Son impressionnante culture sociologique et linguistique lui permet de mobiliser les éléments théoriques et méthodologiques dont elle a besoin pour être à la mesure de la complexité de son objet.

Ce travail constitue ainsi une véritable recherche interdisciplinaire, selon les termes de l'auteure elle-même qui distingue l'«interdisciplinarité», qui intègre diverses disciplines dans une totalité, et la "multidisciplinarité », qui applique séparément différentes disciplines sans les modifier. Approches ethnographique et discursive sont intimement mêlées. Mais cela ne va pas sans risques, comme le souligne Ruth Wodak elle-même dans sa conclusion :

On the one hand, interdisciplinarity opens up new perspectives and allows for novel ideas and innovative approaches; on the other hand, one risks accusations of superficiality if viewed from narrow disciplinary perspectives. (p. 194)

Mais à considérer comment est exposée cette alternative, il ne fait aucun doute qu'elle a opté résolument pour le risque de l'interdisciplinarité, et non pour les «étroites» perspectives des disciplines... De fait, un linguiste ou un analyste du discours peut se sentir frustré, surtout quand il sait que le livre a été écrit par une linguiste de renom. Les analyses strictement linguistiques ne 
sont pas détaillées et l'on ne trouve pas, comme pourrait l'attendre un spécialiste d'analyse du discours, de discussions sur les genres de discours, l'oralité, la textualité, etc. Mais le résultat le plus remarquable de ce travail est justement que les spécialistes des sciences du langage comme les spécialistes des sciences sociales prennent conscience de l'autre face de la réalité qu'habituellement leurs démarches respectives ne leur permettent pas d'appréhender. Des catégories telles que l'identité ou les rapports de pouvoir, par exemple, ne sont pas acceptées comme des présupposés stables mais se voient ainsi travaillées en termes discursifs, appréhendées dans l'épaisseur des interactions verbales.

Si l'on considère les choses du point de vue de la recherche française sur le discours politique, un tel travail apparait extrêmement stimulant, par la vertu qu'il a de décloisonner les espaces. Encore faut-il garder la mesure : le problème n'est pas de décloisonner pour décloisonner, mais de le faire quand c'est nécessaire. Or le type de questionnement dans lequel s'inscrit Wodak, le type de phénomène qu'elle aborde est inaccessible à l'intérieur des frontières disciplinaires. La relation entre «ordre » et « désordre», celle entre frontstage et backstage, celle entre activités réelles au quotidien et fiction médiatique sont une réalité trop fuyante et trop complexe pour se satisfaire d'approches traditionnelles. À partir du moment où l'on admet que le quotidien des parlementaires constitue un élément crucial pour comprendre l'activité politique, le "missing link", pour reprendre une expression de Ruth Wodak, il faut bien se donner les moyens de l'appréhender.

On le voit, la lecture de ce livre ne peut qu'être précieuse pour ceux qui s'intéressent au discours politique, dans la mesure où il témoigne de manière exemplaire d'un tournant heuristique. Les recherches qui s'inspirent de l'analyse du discours «à la française » ont tout intérêt à se confronter à ces problématiques dont l'intérêt ne fait aucun doute.

Dominique Maingueneau

Université Paris 12 Val-de-Marne

maingueneau@univ-paris12.fr

\section{Références}

ABÉLÈs Marc, 1992, La vie quotidienne au Parlement européen, Paris, Hachette.

BELLIER Irène, 1993, L'ENA comme si vous y étiez, Paris, Le Seuil.

LAVE Jean, Wenger Étienne, 1991, Situated Learning. Legitimate Peripheral Participation, Cambridge, Cambridge University Press.

Muntigl Peter, Weiss Gilbert, Wodak Ruth, 2000, European Union Discourses on Un/

Employment : an Interdisciplinary Approach to Employment Policy-Making and

Organisational Change, Amsterdam, Benjamins. 


\section{Actualité bibliographique. Les langages du politique}

Ablali Driss, Ducard Dominique éd., 2009, Vocabulaire des études sémiotiques et sémiologiques, Besançon, Presses universitaires de Franche-Comté, 312 p.

AuSSANT Émilie, 2009, Le nom propre en Inde. Considérations sur le mécanisme référentiel, Lyon, ENSÉditions, $166 \mathrm{p}$.

BADIR Sémir, DUCARD Dominique, 2009, Roland Barthes en cours (1977-1980) : un style de vie, Dijon, Éditions universitaires de Dijon, $160 \mathrm{p}$.

BÉLAND Martine, DutrisAc Myrtô éd., 2009, Weimar ou l'hyperinflation du sens, Laval, Presses de l'université Laval, $227 \mathrm{p}$.

BRUN Josette éd., 2009, Interrelations femmes-médias dans l'Amérique française, Laval, Presses de l'université Laval, 254 p.

Buton François, 2009, L'administration des faveurs. L'État, les sourds et les aveugles (1789-1885), Rennes, Presses universitaires de Rennes, 334 p.

CAMPA Riccardo, 2009, Langage et stratégie de communication, Paris, L'Harmattan, $180 \mathrm{p}$.

CABIN Philippe, DoRTIER Jean-François, 2009, La communication. État des savoirs, Auxerre, Sciences Humaines, $415 \mathrm{p}$.

CHILTON Paul, 2006, Analysing Political Discourse. Theory and Practice, New York, Routledge, $240 \mathrm{p}$.

COHEN Évelyne, 2009, La télévision sur la scène du politique. Un service public pendant les Trente Glorieuses, Paris, L'Harmattan, 204 p.

DACHEUX Éric éd., 2009, Les sciences de l'information et de la communication, Paris, CNRS, $182 \mathrm{p}$.

Debouzy Marianne, 2009, Le monde du travail auxÉtats-Unis. Les temps difficiles (19802005), Paris, L'Harmattan, 292 p.

Delay Christophe, Frauenfelder Arnaud, Pigot Nathalie, Schultheis Franz, 2009, Les classes populaires aujourd'hui. Portraits de familles, cadres sociologiques, Paris, L'Harmattan, $170 \mathrm{p}$.

Le discours et la langue, 2009, t. I (1), Revue de linguistique française et d'analyse du discours, Ethnotypes et sociotypes. Normes, discours, cultures, L. Calabrese, L. Rosier éd., Bruxelles, ULB.

Dracius Suzanne, SAmLong Jean-François, ThÉOBALd Gérard, 2009, La crise de l'OutreMer français. Guadeloupe, Martinique, Réunion, Paris, L'Harmattan, $170 \mathrm{p}$.

Dumons Bruno, Pollet Gilles éd., La fabrique de l'honneur. Les médailles et les décorations en France, $X I X X^{e}-x x^{e}$ siècles, Rennes, Presses universitaires de Rennes, $240 \mathrm{p}$.

DURAMPART Michel éd., 2009, Sociétés de la connaissance. Fractures et évolutions, Paris, CNRS, $182 \mathrm{p}$. 
EkAmbo Jean-Chrétien, KiYındou Alain, Miyouna Ludovic-Robert éd., 2009, Communication et dynamiques de globalisation culturelle, Paris, L'Harmattan, $330 \mathrm{p}$.

ESqUENAZI Jean-Pierre, 2009, La vérité de la fiction. Comment peut-on croire que les récits de fiction nous parlent sérieusement de la réalité?, Paris, Hermès Lavoisier, $208 \mathrm{p}$.

Études françaises, 2008, $\mathrm{n}^{\circ}$ 3, vol. 44, Microrécits médiatiques. Les formes brèves $d u$ journal, entre médiation et fiction, M.-E. Thérenty et G. Pinson éd., Montréal, Presses universitaires de Montréal, $172 \mathrm{p}$.

GAIOTTI Florence, 2009, Expériences de la parole dans la littérature de jeunesse contemporaine, Rennes, Presses universitaires de Rennes, $302 \mathrm{p}$.

GUILLEMETTE Lucie, HÉB ERT Louis éd., 2009, Intertextualité, interdiscursivité et intermédialité, Laval, Presses de l'université Laval, $512 \mathrm{p}$.

GUILLOREL Hervé éd., 2008, Toponymie et politique. Les marqueurs linguistiques du territoire, Bruxelles, Bruylant, $234 \mathrm{p}$.

Harel Simon, 2009, Espaces en perdition. Humanités jetables, t. II, Laval, Presses de l'université Laval, $301 \mathrm{p}$.

Havu Éva éd., 2009, Langues et identités finlandaises, Paris, L'Harmattan, 176 p.

Huck Dominique, KAHN René éd., 2009, Langues régionales, cultures et développement. Étude de cas en Alsace, Bretagne et Provence, Paris, L'Harmattan, 328 p.

LEFEBVRe Rémi, Rog ER Antoine éd., 2009, Les partis politiques àl'épreuve des procédures délibératives, Rennes, Presses universitaires de Rennes, $222 \mathrm{p}$.

LITS Marc éd., 2009, Populaire et populisme, Paris, CNRS, 182 p.

Maingueneau Dominique, 2009, Les termes clés de l'analyse du discours, Paris, Le Seuil, $143 \mathrm{p}$.

Mebiame-Akono Pamphile, 2009, De la linguistique aux sciences du langage. Évolution théorique d'une discipline, Paris, L'Harmattan, $154 \mathrm{p}$.

MENDOZE Gervais, 2009, Insécurité linguistique et appropriation du français en contexte plurilingue, Paris, L'Harmattan, $388 \mathrm{p}$.

MFoutou Jean-Alexis, 2009, La langue de la nourriture, des aliments et de l'art culinaire au Congo-Brazzaville, Paris, L'Harmattan, $236 \mathrm{p}$.

NOYER Jacques, 2009, Quand la télévision donne la parole au public. La médiation de l'information dans «L'Hebdo du médiateur », Lille, Presses universitaires du Septentrion, $326 \mathrm{p}$.

OLIVEIRA Isabelle, 2009, Nature et fonctions de la métaphore en science. L'exemple de la cardiologie, Paris, L'Harmattan, $220 \mathrm{p}$.

Pineira-TREsmontant Carmen éd., 2008, La présidentielle 2007 au filtre des médias étrangers, Paris, L'Harmattan, $386 \mathrm{p}$.

PuÉPI Bernard, 2009, Chroniques des pratiques politiques au Cameroun, Paris, L'Harmattan, $326 \mathrm{p}$.

ReY Alain, 2009, L'esprit des mots, Rouen, Publications des universités de Rouen et du Havre, $48 \mathrm{p}$.

RIVIALE Philippe, 2009, La parole des prophètes. De la Tora à Simone Weil et Gracchus Babeuf, Paris, L'Harmattan, $262 \mathrm{p}$.

Semen, 2009, ${ }^{\circ} 27$, Critical Discourse Analysis 1. Les notions de contexte et d'acteurs 
sociaux, A. Petitclerc et P. Schepens éd., Besançon, Presses universitaires de Franche-Comté, $200 \mathrm{p}$.

Simonnot Brigitte, GALLEzot Gabriel éd., 2009, L'entonnoir. Google sous la loupe des chercheurs en sciences de l'information et de la communication, préface de $\mathrm{H}$. Le Crosnier et en guise de postface, la nouvelle inédite «EnGooglés», de C. Doctorow, Caen, C \& F, 248 p.

TRACOL Matthieu, 2009, Changer le travail pour changer la vie? Genèse des lois Auroux, 1981-1982, préface de O. Wieviorka, Paris, L'Harmattan, 248 p.

Wodak Ruth, 2009, The Discourse of Politics in Action. Politics as Usual, New York, Palgrave McMillan, $256 \mathrm{p}$.

Wolton Dominique, 2009, Informer n'est pas communiquer, Paris, CNRS, 152 p.

Josette Lefèvre

Université de Picardie Jules Verne, CNRS (CURAPP)

josette.lefevre@u-picardie.fr 